Article

\title{
Internal Coordinated Development of China's Urbanization and Its Spatiotemporal Evolution
}

\author{
Liangzhen Zang ${ }^{1,2, *}$ and Yiqing $\mathrm{Su}^{1,2}$ \\ 1 School of Public Policy and Management, Tsinghua University, Beijing 100084, China; \\ syq_005@mail.tsinghua.edu.cn \\ 2 China Institute for Rural Studies, Tsinghua University, Beijing 100084, China \\ * Correspondence: zangliangzhen@mail.tsinghua.edu.cn; Tel.: +86-010-6279-6949
}

Received: 1 November 2018; Accepted: 18 January 2019; Published: 24 January 2019

\begin{abstract}
High urbanization quality, predominantly determined by the degree of internal coordinated development, is the most important factor in promoting urbanization development. Based on the panel data of 283 Chinese cities from 2007 to 2016, this paper analysed the internal coordination degree, spatial distribution and spatiotemporal evolution of urbanization using the methods of range standardization, entropy, and coupling and coordination models, as well as exploratory spatial data analysis. We found the following results: (1) The internal coordination degree of China's urbanization was at a low level for a long time, but it presented a gradually increasing trend; (2) The internal coordinated development of urbanization exhibited an obvious spatial agglomeration. Specifically, it displayed a stepped pattern with a higher internal coordination degree in eastern China, a lower degree in western China, and a spatial distribution with multi-centre agglomeration and diffusion. (3) The spatial distribution of the internal coordinated development of urbanization was stable in most regions of China, only changing in a few. (4) The growth rate of the internal coordination degree of China's urbanization presented the pattern of a dotted distribution, while the growth rate in western China was higher than in central and eastern China. The spatiotemporal evolution relates to the policies changes of China's urbanization. In particular, the urbanization in China transfers its focus from population transfer to the development of quality from 2007 to 2016 . To promote the sustainable and healthy development of China's new urbanization, Beijing-Tianjin-Hebei Region, Yangtze River Delta and Pearl River Delta shall focus on accelerating the transformation of economic development mode; The provinces located around the above three regions shall strengthen the upgrading and promotion of basic public services. The northeast and central provinces shall speed up the innovation of systems and mechanisms and gradually release the potential of urbanization development by promoting the mobility of urban population, and the vast majority of provinces in Western China shall further improve the spatial development potential for urbanization.
\end{abstract}

Keywords: urbanization; internal coordination degree; spatial distribution; spatiotemporal evolution; China

\section{Introduction}

Since the implementation of the Reform and Opening Up in 1978, China's urbanization has made tremendous achievements, such that the urbanization rate has increased from $17.92 \%$ in 1978 to $58.52 \%$ in 2017, with an average annual growth of $1.04 \%$. The process of China's urbanization development may be divided into four stages from 1978 to now. The first stage was from 1978 to 1991, which coincides with the beginning stage of China's Reform and Opening up. In order to promote the development of the rural economy, the government developed urbanization focusing on the expansion of small and medium-sized towns by measures such as encouraging local and nearby 
employment for rural inhabitants. The second stage was from 1992 to 2001, which coincides with the initial stage of China's market economy. This stage witnessed soared economic development of the eastern coastal areas of China. Economic development craved a labor force, for which the government issued a series of related policies to promote the flow of rural surplus labor to large and medium-sized cities, especially to those in the eastern coastal areas. Therefore, China's urbanization scale further expanded, but at the same time, the disparity of urbanization between eastern and western regions has begun to widen. The third stage was from 2002 to 2011, which coincides with a period of rapid development of China's market economy. Although China's urbanization at this stage was guided under a direction of coordinated development among large, medium and small cities, in practice, the disparity of urbanization among different regions has gradually widened due to the pursuit of the expansion of large cities. The fourth stage was from 2012 to present, which coincides with China's fully deepening reforms. Since China's urbanization has been developing in an unbalanced, uncoordinated or unsustainable manner for a long time, this stage targeted high-quality urbanization development. On the whole, formerly government-led urbanization development has gradually come to be dominated by the market, and the market has played a decisive role throughout the process of urban development in China.

However, although China has already entered a the stage of urban society from the perspective of urban population, there are still a number of problems and contradictions in the process of urbanization development [1,2], such as the inconsistency between population migration and land use, conflict between urban layout and scale structure, the contradiction between economic development and environment protection, and unbalanced public services between urban and rural areas [3,4]. In fact, these issues result from weak internal coordinated development. Urbanization goes beyond population, economy or space, and includes such factors as public services, ecological environment, and so forth [5]. With urbanization covering various aspects, scholars now, taking a holistic view, argue that urbanization mainly consists of subsystems including population, economy, public services, ecology and space, and so forth [6-8]. Therefore, internal coordinated development of urbanization is a complex system. Internal coordinated development of urbanization implies the coordinated development of various subsystems such as the economy, public services and population. In other words, it aims to maintain a coordination relationship, and achieve the coordinated development of each and every subsystem inside the urbanization system through the interaction of these subsystems, so as to push forward the steady development of urbanization. If one of the subsystems is seriously backward compared with the others, it will lead to the unbalanced and unsustainable development of urbanization, which will seriously restrict the overall development thereof. Taking a certain region for an example, if it boasts a high development level in terms of economy, public services, and urban scale, but its quality of ecological environment is poor, its internal system is out of tune and it can be demonstrate weak internal coordination in terms of urbanization.

Currently, there is unbalanced development in population urbanization across all of the cities in China [9]. If the disparity of internal coordinated development of China's urbanization continues to widen, it will result in more social and economic problems. Therefore, it is urgent to explore the internal coordinated development and the spatiotemporal evolution of China's urbanization. The purpose of this paper is to evaluate the degree of interaction among urbanization internal subsystems, that is, the internal coordination degree of urbanization, on the basis of building an indicator system of internal coordinated development of urbanization. Furthermore, it analyses the spatial and temporal evolution for the internal coordination degree of urbanization in various cities from the perspective of areal distribution. This research could provide a corresponding theoretical guide for the creation of a macro-urbanization policy in China, also recommending policies that will improve the quality of urbanization in the future. 


\section{Literature Review}

\subsection{Coordination Between Urbanization and Economic Development}

Previous research showed that there was a coordination relationship between urbanization and economic development. On the one hand, a number of researchers believed that urbanization can significantly promote economic development [10-12]. For instance, Arvin found that the continuous development of urbanization played an important role in promoting the economy in the G20 from 1996 to 2012 [13]. Bao found that the comprehensive contribution rate of urbanization to economic growth was as high as 30\% from 1997 to 2011 in China [14]. Furthermore, researchers have also insisted that the effect of urbanization on the economy exhibited a characteristic of spatial distribution because of regional differences. The economy will grow at $0.2 \%$ if there is $1 \%$ growth of urbanization in regions of China with middle and high income, while there is no effect in the lower-income regions [15].

On the other hand, many researchers who discussed the impact of economic development on urbanization believed that rapid economic development had a great influence on promoting urban development. For instance, Park's study on the Mongolian Plateau [16] and Wang's study on China's Dongting Lake Region [17] confirmed the above viewpoint. In contrast, only a few scholars argued that urban and economic development were exclusive to each other. Brückner found that there was a negative effect on the per capita GDP growth with the increasing urbanization rate across 41 African countries from 1960 to 2007 [18].

\subsection{Coordination Between Urbanization and Ecological Environment}

With the gradual transformation of human activities to non-farming industries, urbanization is directly or indirectly affecting the entire ecological environment [19-23]. Three viewpoints were exhibited by previous research. First, the coordination relationship between urbanization and the ecological environment has improved gradually with the labour mitigation from village to city all over the world in recent years; i.e., there was a trend from a seriously unbalanced stage to a highly balanced stage [24]. In China, there was a gradually improving trend, although the coordination relationship between urbanization and the ecological environment changed tortuously slowly. For example, the coordination relationship in the Beijing-Tianjin-Hebei region and the Yangtze River Delta displayed an S-shaped curve [25,26], and the Huaibei region showed an inverted U-shaped curve [27]. Second, some researchers argued that rapid urbanization development could result in an over-concentration of resources that could deteriorate the ecological environment to a large extent [28-30], such as by reducing biodiversity [31]. Third, a number of researchers believed that the relationship varied in different regions [32,33]. Taking the coordination relationship between urbanization and PM 2.5 as an example, PM 2.5 levels displayed a downward trend when the urbanization rate was increasing in Europe and Latin America but exhibited an opposite trend in East Asia, Southeast Asia, South Asia and Africa [34].

\subsection{Coordination Between Urbanization and Land Use}

The existing research mainly discussed the coordination relationship between urbanization and land use from the perspectives of the temporal and spatial trends [35-37]. Recent studies hold that the relationship was gradually improving over time [38]. In China, it varied from a low-coordinated stage to a medium-coordinated stage, making great progress since 2012 when the Promoting the Development of New Urbanization Plan was carried out [39]. In addition, researchers insisted that there was spatial inequality for the coordination relationship between urbanization and land use. For instance, the regions with a higher coordination degree were mainly located in the eastern and central regions of China, but they presented a completely different pattern [40]. Examples include the pattern of urban agglomeration in the Yangtze River Economic Belt [41], the pattern of polycentric clustering in China's northeast region [42], and the pattern of encircling diffusion in the Yunnan Provinces [43]. 


\subsection{Research Review}

According to the above review, it can be seen that research on the coordination relationship between urbanization and other factors has become a focus of attention. However, previous research has mainly focused on population urbanization and less on comprehensive urbanization; i.e., researchers used to take the proportion of urban population as the indicator of urbanization rather than integrated indicators. Therefore, although many data on the coordination relationship have already been analysed, most of these studies explored only the interactional effect between the population and other factors. In addition to the indicator of population, other elements, such as economic development, social progress, spatial structure, resources and the environment, were not considered in the analyses in most of the existing literature. Thus, the internal coordinated development of urbanization was ignored.

Furthermore, the existing research paid less attention to the analysis of regional characteristics of the internal coordinated development of urbanization. Existing studies hold different views on the coordination relationship between urbanization and other factors, because the researchers usually focused on a limited area. Therefore, exploring the spatiotemporal trends of the coordinated development of China's urbanization could further reveal the effect of spatial spillover. However, there was no systematic discussion about the spatiotemporal trends in the existing research. By regarding urbanization as a comprehensive system and dividing it into several subsystems, this paper, focusing on the analysis of the spatiotemporal evolution of internal coordinated development of China's urbanization, can not only complement the theory of Synergetics in the field of urbanization, but can also provide theoretical basis for policy formulation to promote the high-quality development of urbanization in China.

\section{Framework, Method and Data Source}

\subsection{Framework}

The available literature mainly studies the building of indicator system of urbanization from three perspectives. First, an indicator system is built from a global perspective such as the City Development Index and Urban Indicators Guidelines formulated by United Nations Centre for Human Settlements. These two indicator systems can effectively evaluate the interaction of various subsystems of urbanization such as population, economy and public services, but lack pertinence due to their wide range [44]. Second, the indicator system is built from a national perspective such as the indicator system evaluating Japanese urbanization; an example is the UK indicator system put forward by Cloke [45]. It covers three subsystems of population, residence and traffic. Third, the indicator system is built from a perspective of economic development stage, such as the system put forward by Inkeles including subsystems of population, cities and other factors [46].

Many scholars are also engaged in the building of indicator systems in light of the specific conditions of urbanization in China. For example, System of Quality Evaluation of Urbanization of China outlines four subsystems including low carbon urbanization, harmonious urbanization, happy urbanization and sensible urbanization with nearly 300 indices [47]. Research on Quality Evaluation and Promotion Path of Urbanization puts forward an indicator system of three subsystems of quality, efficiency promotion, and coordination between rural and urban areas [48]. The Team In-Chief of Research on Cities in China proposes an indicator system composed of subsystems of social development and ecological environment [49]. Moreover, scholars like Zhang [50], Lv [51], and Fang [52] also bring up with their own research. In general, the indicator systems above mainly are composed of five aspects, i.e., economy, public services, population, space and ecology.

In terms of economy, economic growth is an important driving force for urbanization. On the one hand, it drives production forces to flow to cities constantly, increasing their input and output and generating economies of scales. On the other hand, it breeds more sophisticated industrial structures. With regard to public services, it pushes forward equality in basic services, realizing the common 
development of healthcare, education, social security and transport, which are important issues in the urbanization in China. As for population, the higher density of urban population has become the most typical phenomenon during the urbanization, along with the change of working population of the tertiary industry and urban unemployment rate, due to the flow of population from countryside to cities. In the aspect of space, during urbanization, the land usage shifts from agricultural land to the non-agricultural one and the location and scenery are expanding in the process. With respect to ecology, the ecological environment, including both ecological environment foundation and protection, serves as an important carrier for the production and living of urban residence. A good ecological environment is the crucial safeguard for sustainable development of urbanization.

In the 1970s, Harmann Haken, a German scientist, came up with Synergetics, which studies how to present the ordered structure at time, space, and function levels through internal synergistic effect, in the context of material or energy interchange between the open system with unbalanced condition and the external environment $[53,54]$. Synergy theory holds that there is a relationship of interaction and cooperation among various subsystems in the whole system; it is usually used to analyse how the complex system structure could be ordered $[55,56]$. That is, it studies how to use the interaction among each subsystem to exert the overall effect of the system. Synergetics offers important theoretical support for the study of internal coordinated development of urbanization. First, as for the application range, urbanization is an open system, which continuously makes elements exchange with the external environment, so that the internal coordinated development system of urbanization is in line with the application conditions of Synergetics. Second, the internal coordinated development system of urbanization includes many subsystems such as population, economy and public services, and these subsystems correlate and interact with each other, influencing the overall effect. Third, the change of external variables of urbanization internal coordinated development system, such as information flow, material flow and energy flow, will effectively exert the overall synergistic effect of this system, achieving higher level of synergy. Specifically, the relations among subsystems of the urbanization system are as follows:

First, space is the foundation for the interaction of the population, economy, public services and ecology. The changes of these four elements usually take place based on the urban space. However, space has some characteristics because it needs to follow natural rules, such as the distribution of natural resources, the change of population flow, etc. Therefore, the urbanization that is in line with spatial evolution characteristics can promote the interaction of all elements effectively. Second, the population is the driving force that promotes the interaction of the elements. The phenomenon of a continuous population influx from the countryside into the city could greatly stimulate investments and consumption and promote economic development. Furthermore, the city space continues to expand, and the spatial structure continues to change in order to meet the space demand caused by the population agglomeration, which also plays an important role in improving infrastructure and deteriorating the ecological environment [57]. Third, economic development could affect the whole system structure. With gradual economic development, urban public services could be improved in order to attract the rural population to the city. Moreover, cities' resources are usually over-consumed and over-utilized along with the variation of urban spatial structure and the ecological environment [58]. Fourth, the continuous improvement of infrastructure could gradually influence other elements. Along with urban space expansion, the improved infrastructure provides a good foundation for urban economic development that drives the population agglomeration to the city [59]. Finally, ecological environment lays a good foundation for healthy urbanization development. The development of the economy, population, public services and space cannot go beyond the capacity of the ecological environment [60].

From the perspective of qualitative analysis, the interrelationship among the five subsystems of urbanization system, economy, public services, population, space and ecology is explained clearly, and we find that there is a direct two-way mechanism of impact among the economy, public services, population, ecology and space. In order to analyse the temporal trend and spatial distribution of 
internal coordinated development as a whole, this paper integrated and expanded the above five subsystems, and established the quantitative indicators. Then, a discussion could be made from a qualitative perspective on the basis of econometric results. Therefore, it is important to choose quantitative indicators. According to the literature that took the quantitative indicators to analyse urbanization $[2,4,9,14,20,46-52,61-65]$, we built an internal coordinated development indicator system including 23 indicators based on the indicators in the China's National Plan on New Urbanization (2014-2020) and the indicator representativeness and data availability (Table 1).

Table 1. Internal coordinated development indicator system of urbanization.

\begin{tabular}{|c|c|c|c|}
\hline System & Indicator & System & Indicator \\
\hline \multirow{6}{*}{ Economy } & Per capita GDP & \multirow{3}{*}{ Population } & Population density \\
\hline & Proportion of the tertiary industry & & Proportion of tertiary industry employees \\
\hline & Government revenue & & Registered urban unemployment rate \\
\hline & Fixed asset investment & \multirow{3}{*}{ Space } & $\begin{array}{l}\text { Proportion of built-up area to the total area } \\
\text { of the area }\end{array}$ \\
\hline & Total retail sales of consumer goods & & Per capita urban road area \\
\hline & $\begin{array}{l}\text { Proportion of technology expenditure in } \\
\text { fiscal expenditure }\end{array}$ & & Per capita green area of public places \\
\hline \multirow{6}{*}{$\begin{array}{l}\text { Public } \\
\text { Services }\end{array}$} & Number of hospitals & \multirow{6}{*}{ Ecology } & Wastewater discharge \\
\hline & Number of primary and secondary schools & & Sulfur dioxide emissions \\
\hline & Number of public buses & & Emission of smoke and dust \\
\hline & Number of public post offices & & Recovery rate of industrial solid waste \\
\hline & $\begin{array}{l}\text { Number of public books owned by every } \\
\text { hundred people }\end{array}$ & & Green coverage rate in built-up area \\
\hline & Number of internet users & & \\
\hline
\end{tabular}

\subsection{Method}

In order to study the spatiotemporal evolution status of internal coordinated development of urbanization in China, this paper first undertakes standardized processing of indicator system with Range Normalization Method, and then uses the Entropy Method to calculate development degree of the economy, public services, population, ecology and space. Afterwards, the Coupling and Coordination Model is used to calculate the coupling and coordination degree of internal coordinated development of urbanization in China, and the coupling and coordinated development status of urbanization internal subsystems of 283 cities is acquired. Finally, the Exploratory Spatial Data Analysis is used to study the regional spatiotemporal evolution status of internal coordinated development of urbanization in 283 cities from the perspective of spatial and temporal evolution.

\subsubsection{Range Normalization Method}

Based on the data, we built the matrix $X=\left(x_{i j}\right)_{m \times n}, i=1,2 \ldots \ldots, n, j=1,2 \ldots \ldots, m$, where $x_{i j}$ is the $j$-th indicator of $i$-th City, $m$ is the total indicator numbers, and $n$ is the city numbers. The range normalization method is then used to standardize the initial data because the dimensions, units and attributes of all of the indicators in the indicator system are different.

$$
\begin{aligned}
x_{i j}^{\prime} & =\frac{x_{i j}-\min x_{j}}{\max x_{j}-\min x_{j}}(\text { If the data are positive }) \\
x_{i j}^{\prime} & =\frac{\max x_{j}-x_{i j}}{\max x_{j}-\min x_{j}}(\text { If the data are negative })
\end{aligned}
$$

where $\max x_{j}$ and $\min x_{j}$ are the maximum and minimum values of the $j$-th indicator, respectively. Then, the new matrix, $X_{i j}^{\prime}=\left(x_{i j}^{\prime}\right)_{m \times n}$, is built by using the standardized data. 


\subsubsection{Entropy Method}

Originally used in physics, Entropy is a concept from the field of thermodynamics that is mainly used to reflect the degree of chaos within the system. In 1948, the concept of entropy was introduced into information theory by Shannon [66]. The entropy method can be used to analyse the discrete degree of each indicator in multi-index system and calculate the comprehensive evaluating value of the system. The core of the entropy method is to determine the index weight of each indicator by highlighting the indicator differences within the system. If there is a greater discrete degree for an indicator, it means that this indicator has a greater impact on the multi-index system. Therefore, according to the discrete degree of each indicator, the weight of each indicator can be calculated, and finally, the comprehensive evaluation degree will be calculated. The Entropy method has already been used for analysis in a lot of fields, such as economy [67], environment [68,69], urbanization [70] or comprehensive fields [71].

In order to estimate the internal coordinated development of China's urbanization, the development degree of the economy, public services, population, ecology and space need to be calculated. Because each subsystem contains a lot of indicators, in this paper, we used an entropy method that could reflect the importance of each indicator in the indicator system. At the beginning, all the standardized data will be formed into matrix $X^{\prime}$. Then, the entropy $e_{j}$ corresponding to the $j-t h$ indicator in the matrix $X^{\prime}$ is calculated by:

$$
\begin{gathered}
e_{j}=-\frac{1}{\ln m} \sum_{i=1}^{m} p_{i j} \ln p_{i j} \\
p_{i j}=x_{i j}^{\prime} / \sum_{i=1}^{m} x_{i j}^{\prime}, i=1,2, \ldots, n ; j=1,2, \ldots, m
\end{gathered}
$$

The formula for the entropy weight is:

$$
w_{j}=\left(1-e_{j}\right) / \sum_{j=1}^{n}\left(1-e_{j}\right)
$$

The comprehensive evaluation function of urbanization of $i$-th city in $t$-th year is:

$$
f_{i}\left(U_{t}\right)=\sum_{j=1}^{m} W_{j t}^{\prime} y_{i j t}^{\prime}
$$

Similarly, the development degree of the economy, public services, population, ecology and space can also be calculated by: $f_{i}\left(U_{k t}\right), k=1,2 \ldots \ldots, 5$.

\subsubsection{Coupling and Coordination Model}

This paper uses the internal coordination degree to evaluate the degree of internal coordinated development of urbanization in China. The internal coordination degree should be calculated on the basis of coupling degree that is calculated by the following formula:

$$
C=\frac{5 \sqrt[5]{f_{i}\left(U_{1 t}\right) \times f_{i}\left(U_{2 t}\right) \times f_{i}\left(U_{3 t}\right) \times f_{i}\left(U_{4 t}\right) \times f_{i}\left(U_{5 t}\right)}}{f_{i}\left(U_{1 t}\right)+f_{i}\left(U_{2 t}\right)+f_{i}\left(U_{3 t}\right)+f_{i}\left(U_{4 t}\right)+f_{i}\left(U_{5 t}\right)}
$$

where $C$ is the coupling degree that represents the interaction ability of the economy, public services, population, ecology and space. Generally, the value of $C$ is between 0 and 1 . This indicates that there is a strong interaction if $C$ tends towards 1 and a weak interaction if $C$ tends towards 0 .

However, from the coupling degree, it is impossible to judge whether the subsystems are related at a higher or lower level, although it can reflect whether there is close relationship among all the subsystems. For example, if the development degree of each subsystem in urbanization is a low level, the coupling degree is also higher. However, this does not mean that there is a coordinated relationship. Thus, the coordination model is introduced as follows:

$$
D=\sqrt{C \times T}
$$


where $D$ is the coordination degree and $T$ is the comprehensive coordination index. The range of $D$ is similar to $C$. When $D$ is equal to 1 or 0 , it means that the five elements are coordinated or uncoordinated, respectively. Generally, when $0<\mathrm{D} \leq 0.3$, it indicates that they are in a weak coordination level; when $0.3<\mathrm{D} \leq 0.5$, it indicates that they are in a weaker-to-medium coordination level; when $0.5<\mathrm{D} \leq 0.8$, they are in a medium-to-high coordination level; $0.8<\mathrm{D} \leq 1$, they are in a high coordination level. $D$ will close to 1 when the five subsystems infinitely developed in a synchronous manner.

The comprehensive coordination index $T$ can be calculated by the following formula:

$$
T=\sum_{k=1}^{5} \alpha_{k} f_{i}\left(U_{k t}\right)
$$

where the sum of $\alpha_{k}$, the contribution of five elements, is equal to 1 . This paper argues that there is the same contribution from the five elements in the urbanization indicator system. Here, we set $\alpha_{k}$ as 0.2 .

\subsubsection{Exploratory Spatial Data Analysis}

Global spatial autocorrelation analysis and hot spot analysis are used in this paper in order to explore the spatial correlation of the coupling and coordination degrees of five elements of urbanization. Global spatial autocorrelation can be calculated by the following formula:

$$
\text { Moran's } I=\frac{\sum_{i=1}^{n} \sum_{j=1}^{n} w_{i j}\left(x_{i}-\bar{X}\right)\left(x_{j}-\bar{X}\right)}{S^{2} \sum_{i=1}^{n} \sum_{j=1}^{n} w_{i j}}
$$

where Moran's I is the global spatial autocorrelation index, $w$ is the spatial weight matrix, $X$ is the value

of the attribute, $\bar{X}$ is the mean and $S^{2}$ is the variance. Normally, Moran's I ranges from -1 to 1 . There is no spatial correlation among all the regions when it tends towards 0 and a positive or negative spatial correlation when it tends towards 1 or -1 .

Hot spot analysis is used to explore the high-value or low-value agglomeration state of coordination degree.

$$
G_{i}^{*}(d)=\frac{\sum_{j}^{n} \omega_{i j}(d) Y_{j}}{\sum_{j}^{n} Y_{j}}
$$

After standardized processing of $G_{i}^{*}(d)$, the standard form is:

$$
Z\left(G_{i}^{*}\right)=\frac{G_{i}^{*}-E\left(G_{i}^{*}\right)}{\sqrt{\operatorname{var}\left(G_{i}^{*}\right)}}
$$

where $E\left(G_{i}{ }^{*}\right)$ is the expected value of $G_{i}{ }^{*}$ and $\operatorname{var}\left(G_{i}{ }^{*}\right)$ is the variance. The hot spot zone can be formed according to the value of $Z$.

\subsection{Data Source}

The data used in this paper range from 2007 to 2016, as reported in the China City Statistical Yearbook 2008-2017. This paper selected only 283 Chinese cities as the research focus because there were many missing data for other cities in the yearbook.

\section{Results and Discussion}

\subsection{Temporal Trend of the Coupling and Coordination Degrees}

The curves of the average coupling degree and the coordination degree of urbanization of 283 cities from 2007-2016 are exhibited in Figure 1. As seen in the figure, we found three characteristics. First, 
the coupling and coordination degrees of China's urbanization were at a low level for a long time. Specifically, the average coupling degree, changing between 0.409 and 0.509 , was just 0.458 in a $10-y r$ period, and the average coordination degree, changing between 0.280 and 0.341 , was just 0.314 . Second, the coordination degree was always smaller than the coupling degree. The disparity was 0.144 on average and fluctuated between 0.123 and 0.168 during the 10 -yr period. This result indicated that there was a characteristic of long-term differentiation of internal coordinated development of China's urbanization. Third, the coupling and coordination degrees of urbanization displayed a stable trend. Both of the coupling and coordination curves presented the lowest points in 2007 and the highest points in 2016, with an average annual growth of $2.46 \%$ and $2.23 \%$, respectively.

Overall, the changing trend of China's urbanization coordination was closely related to the relative policies. Before 2014, the policies emphasized population urbanization rather than urbanization quality. However, with the implementation of the China's National Plan on New Urbanization (2014-2020), the comprehensive development of urbanization was considered in order to avoid problems, such as the excessive use of agricultural land, inefficient economic growth, and inadequate investment infrastructure.

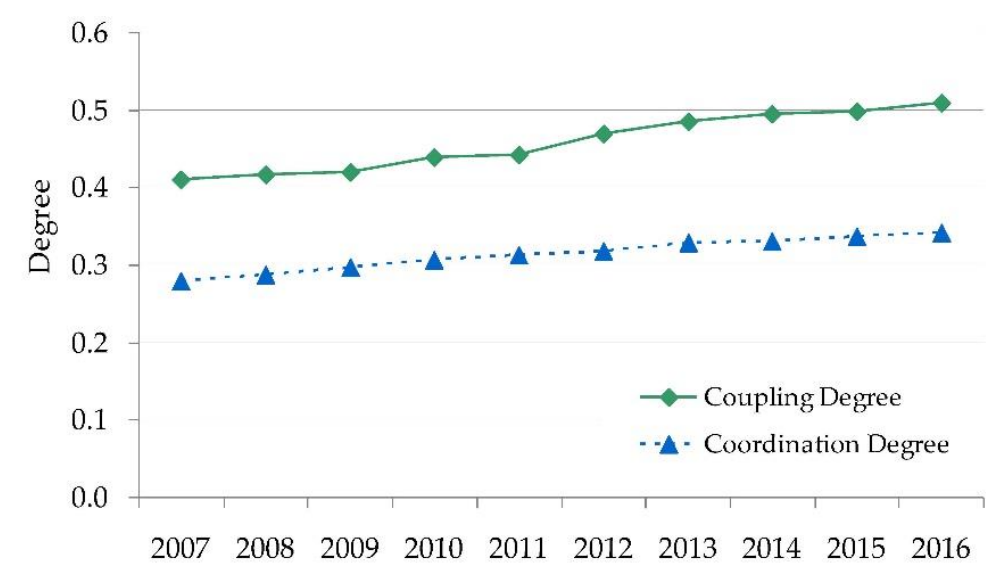

Figure 1. The coupling and coordination degrees in 2007-2016.

\subsection{Analysis on Coordination Types}

By taking 0.1 as the interval, the range of the coordination degree from 0 to 1 was divided into ten types from the lowest to the highest, with the range of type I of $(0,0.1)$. The data from 4 years-2007, 2010, 2013 and 2016 - were entered into the analysis. Table 2 shows the city numbers in each type for the four years.

Table 2. Number of cities in ten types from 2007 to 2016.

\begin{tabular}{ccccccccccc}
\hline Year & I & II & III & IV & V & VI & VII & VIII & IX & X \\
\hline 2007 & 0 & 8 & 195 & 65 & 11 & 2 & 2 & 0 & 0 & 0 \\
2010 & 0 & 0 & 167 & 89 & 22 & 3 & 1 & 1 & 0 & 0 \\
2013 & 0 & 0 & 124 & 115 & 33 & 7 & 2 & 2 & 0 & 0 \\
2016 & 0 & 0 & 98 & 136 & 34 & 10 & 3 & 2 & 0 & 0 \\
\hline
\end{tabular}

We found that there were two characteristics of China's coordination. First, there were few cities in each type with higher or lower coordination degrees. Specifically, there were no cities in types I, II, IX and X within the reported years, except for 8 cities in type II in 2007. Comparatively, most cities were in type III and type IV, ranging from 0.2 to 0.4 . The proportions of these two types in all 283 cities over the four study years were $91.87 \%, 90.46 \%, 84.45 \%$ and $82.69 \%$, respectively. Second, the numbers in the types with higher coordination degrees increased from 2007 to 2016. Specifically, the numbers in type III decreased, while the numbers in types IV, V and VI increased gradually. This result 
indicated that the joint improvement of internal coordinated development of urbanization in most cities contributed to an increase of the average coordination degree. Furthermore, it also denoted that a long period is needed to achieve urban development with joint improvement of the economy, public services, population, ecology and space.

\subsection{Spatial Autocorrelation Analysis}

Table 3 shows the Moran's I of the coupling and coordination degrees that explained the spatial autocorrelation of the 283 cities from 2007 to 2016.

As seen in Table 3, each Moran's I is greater than 0 and passes the significance test at $1 \%$ during the 10-yr period. This finding indicated that a positive spatial correlation existed across all of the cities, and there was a local agglomeration in the space. In other words, the findings presented a spatial structure of binary distribution; i.e., cities with higher coupling and coordination degrees agglomerate each other in the process of urbanization development, and those with lower degrees displaying a similar distribution.

Moreover, the spatial agglomerative effect of the coupling and coordination degrees of China's urbanization was gradually increasing. The Moran's I of the coupling degree was just 0.196 in 2007 and reached 0.228 in 2016, while the Moran's I of the coordination degree was 0.230 in 2007 and reached 0.260 in 2016. Comparatively, the Moran's I of the coupling degree was always smaller than the Moran's $I$ of the coordination degree throughout the study period. This finding fully demonstrated that the binary distribution among all of the cities was becoming more and more apparent in the process of China's urbanization.

Table 3. Moran's I of the coupling and coordination degrees in 2007-2016.

\begin{tabular}{cccccccccccc}
\hline Degree & Year & $\mathbf{2 0 0 7}$ & $\mathbf{2 0 0 8}$ & $\mathbf{2 0 0 9}$ & $\mathbf{2 0 1 0}$ & $\mathbf{2 0 1 1}$ & $\mathbf{2 0 1 2}$ & $\mathbf{2 0 1 3}$ & $\mathbf{2 0 1 4}$ & $\mathbf{2 0 1 5}$ & $\mathbf{2 0 1 6}$ \\
\hline \multirow{3}{*}{ Coupling } & Moran's I & 0.196 & 0.191 & 0.176 & 0.197 & 0.205 & 0.214 & 0.212 & 0.223 & 0.214 & 0.228 \\
degree & Z value & 5.326 & 5.207 & 4.800 & 5.363 & 5.552 & 5.793 & 5.732 & 6.023 & 5.784 & 6.175 \\
& $P$ value & 0.000 & 0.000 & 0.000 & 0.000 & 0.000 & 0.000 & 0.000 & 0.000 & 0.000 & 0.000 \\
\hline \multirow{3}{*}{ Coordination } & Moran's $I$ & 0.230 & 0.226 & 0.202 & 0.228 & 0.227 & 0.228 & 0.229 & 0.230 & 0.244 & 0.260 \\
degree & $Z$ value & 6.292 & 6.192 & 5.541 & 6.227 & 6.198 & 6.221 & 6.245 & 6.628 & 6.661 & 7.071 \\
& $P$ value & 0.000 & 0.000 & 0.000 & 0.000 & 0.000 & 0.000 & 0.000 & 0.000 & 0.000 & 0.000 \\
\hline
\end{tabular}

\subsection{Hot Spot Analysis}

The correlation coefficients of the 283 cities were calculated by using the hotspot analysis method in order to recognize the regional location that contributed the most to the spatial autocorrelation. In this study, we calculated the Getis-Ord $G^{*}$ that was divided into four parts by the Jenks natural breaks optimization. The scatter maps of hot spots in 2007, 2010, 2013 and 2016, including hot spot zone, sub-hot spot zone, sub-cold spot zone and cold spot zone, were generated by ArcGIS (Version 10.2, Environmental Systems Research Institute, United States) (Figure 2).

On the whole, the spatial distribution of the internal coordination degree of China's urbanization exhibited two characteristics. First, it presented a stepped spatial distribution from 2007 to 2016. The regions with higher internal coordination degrees were mainly concentrated in the eastern coastal regions of China, followed by the central regions, while the agglomeration of lower internal coordination degrees was most obvious in the western regions of China. Second, the multi-centre agglomeration diffusion of the internal coordinated development of urbanization was obvious in 2007-2016. The hot spot zone was predominately distributed in three regions: the Beijing-Tianjin-Hebei, the Yangtze River Delta and the Pearl River Delta. Because these three regions were surrounded by the sub-hot spot zone, we can conclude that these three regions could play an important role in promoting the coordinated and balanced development of urbanization to the surrounding regions.

From the perspective of spatiotemporal evolution, the spatial distribution of the internal coordinated development of China's urbanization did not clearly change, with only small changes occurring in a few regions. In terms of the evolution from the hot to the cold zones, the internal 
coordinated development of urbanization in northeast China was gradually weakening over the past ten years, with obvious change from a sub-cold spot zone to a cold spot zone on a large scale. Similarly, Erdos, Xinyang, Suizhou, Xiangyang and their surrounding areas gradually moved into a cold spot zone. The internal coordinated development of urbanization in these regions presented an imbalanced trend. In terms of the evolution from the cold to the hot zones, cities in southwestern Shandong Province, northwestern Jiangsu Province, Urumqi and Karamay in the Xinjiang Autonomous Region shifted from a sub-cold spot zone into a sub-hot spot zone. The internal coordination in these regions strengthened gradually during the 10-yr period.

We found that there are different reasons for the disparities of the internal coordination degree of urbanization across various cities. China's mega-cities, predominately located in Beijing-Tianjin-Hebei, the Yangtze River Delta and the Pearl River Delta, are relatively developed in terms of economy, urban planning, population migration, public services and ecological protection. Although other cities of eastern China had the same situation, such as rapid economic development, large population migration, and urban area expansion, the most important problem of urbanization development was the ecological environment deterioration that lowered the urbanization quality. For the cities in China's central regions, the economic development was mainly promoted by the land expansion that often resulted in ignoring the public services. Because the rapid economic development did not effectively match the supply of social public services, the internal coordinated development of urbanization in central regions was weak compared with that of the eastern regions. Comparatively, the internal coordinated development of urbanization in the cities of the western regions was relatively low because of the lagged economic development.


Figure 2. Hot spots variation maps of internal coordinated development of China's urbanization.

\subsection{Analysis on the Growth Rate of the Internal Coordination Degree of Urbanization}

Although the internal coordination degree of some cities decreased gradually, we found that the average growth rate of the internal coordination degree of urbanization of the 283 cities increased 
during the 10-yr period. Figure 3 shows the quartered map of the average growth rate. It can be seen that the growth rate of the internal coordination degree presented an obvious pattern of a dotted distribution; i.e., the internal coordinated development of urbanization displayed a different growth trend across all of the cities. On the whole, the growth rate of the internal coordination degree of urbanization in western regions was higher than that in central and eastern regions, especially in some cities of Gansu, Xinjiang, Guizhou and Szechwan. In contrast, the growth rate in the cities of northeastern China was relatively lower.

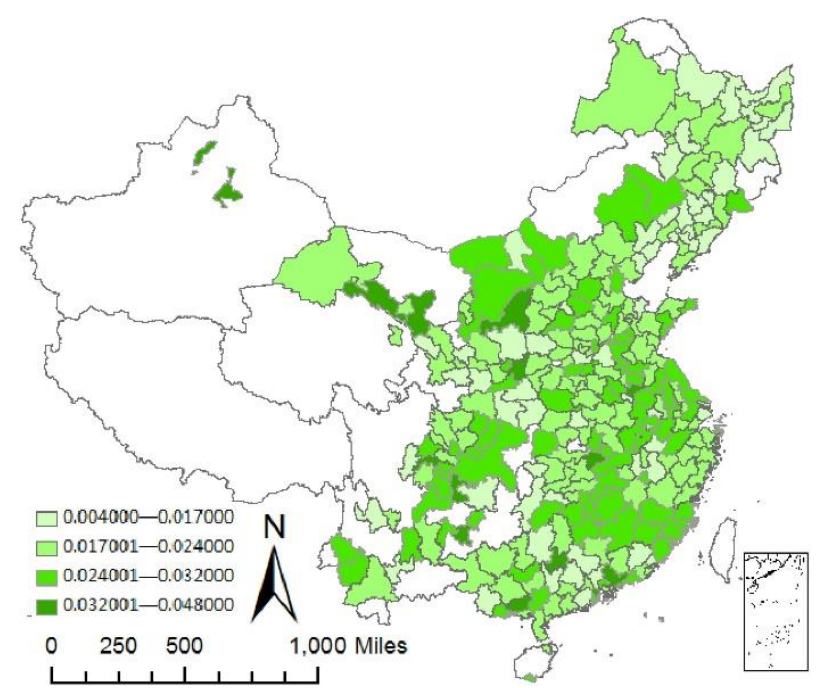

Figure 3. Quartile graph of average growth rate of the internal coordination degree in 2007-2016.

\section{Discussion}

According to the analysis result above, the internal coordinated development of urbanization in China has obvious features of spatiotemporal evolution. Generally, it not only conforms to the general regulations of the urbanization process, but it also relates to the policies of each stage. However, different spatial areal distributions of internal coordinated development of urbanization in China lie in the different growth speeds of each subsystem. According to the connotation transition of urbanization, the urbanization in China transfers its focus from population transfer to the development quality from 2007 to 2016. In 2007, the economic policies in China mainly focused on the development towards large and medium size cities, such as Inform of Developing Surplus Rural Labor Current Employment and Opinions on Developing Transfer Employment of Surplus Rural Labor, the purpose of which is to achieve the economic growth with population transfer to ensure economic development and city expanding of large and medium size cities. The urbanization rate of Chinese population exceeded $50 \%$ for the first time in 2011. Afterwards, the urbanization process in China further emphasized on the decisive role of the market on resource allocation. The internal coordinated development of urbanization has begun to attract attention, and great importance has also been attached to the quality of development. In 2014, China issued the National Plan on New Urbanization (2014-2020) and the Opinions on Further Promoting the Reform of the Household Registration System, which indicated that the urbanization in China had ushered in a new development stage that focuses on the quality of urbanization. Meanwhile, with the introduction of the policies for migrant workers to return home to start businesses and obtain employment and policies to promote nearby and local urbanization in various places (especially in the western regions), the development of urbanization in the western regions has been rapidly accelerated.

The specific policies on such areas as land, population and environment are undergoing change as China gradually transforms its urbanization development goal into focus on the quality of internal coordinated development of urbanization. For example, in the area of education, the Chinese government is constantly exploring and fine-tuning policies on guaranteeing the education of migrant children, so as to gradually solve the problems such as enrollment in different places and educational 
equity. At the same time, the state has also provided substantial support to education in China's western region to solve the problem of unfair distribution of educational resources. In the area of medical care, China began to promote a new rural cooperative medical scheme nationwide in 2006, extended the basic medical insurance system to cities and towns in 2007, established the goal of universal medical insurance in 2009, and gradually achieved comprehensive health insurance coverage. Medical infrastructure has witnessed significant improvement. In the area of land, with the rapid expansion of urbanization and urban space, the Chinese government has successively rolled out relevant policies such as Decision on Deepening Reform of Strict Land Management, Notice on Promoting the Economical and Intensive Use of Land, and Opinions on Strengthening the Protection of Cultivated Land and Improvement, so as to gradually improve the basic system of the market allocation of land resources. In terms of population mobility and employment, China has put more emphasis on the role of the market in the allocation of labor resources during the urbanization development. Policies have been introduced to promote home-bound entrepreneurship and employment. In particular, the state has provided the support for the economic development of the western region, which has narrowed down the differences between urban and rural development in different regions. In terms of ecological environment, China is gradually adapting to the new concepts and trends of modern urban development during its urbanization. It is emphasized that through the construction of green modes of production, lifestyle and consumption, ecological civilization has been fully integrated into urban development over the years in order to promote urban green development.

\section{Conclusions}

Based on the indicator system of the internal coordination degree of China's urbanization, the range normalization method, entropy method, coupling and coordination models and exploratory spatial data analysis were used to analyse the internal coordination degree, spatial distribution and spatiotemporal evolution by using the data of 283 cities from 2007 to 2016 . According to the results of this research, we found the following.

First, the internal coordination degree of China's urbanization has been at a low level for a long time, but it displayed a gradually increasing trend. Although the average internal coordination degree was only 0.314 , while the proportion of cities ranging between 0.2 and 0.4 was greater than $80 \%$ from 2007 to 2016 , the situation of uncoordinated urban development is improving gradually. The improvement in the internal coordinated development of urbanization is not caused by the obvious increase in a few cities. In contrast, the common improvement in the internal coordination degree in a number of cities contributes to the continuous improvement in the average level among the 283 cities.

Second, there is a positive spatial correlation of the internal coordinated development of China's urbanization, and the spatial agglomeration effect was gradually strengthened during the 10-yr period. The internal coordination degree of China's urbanization displayed the spatial distribution of a stepped pattern, with a higher degree in the eastern regions of China and a lower degree in the western regions. In addition, the internal coordinated development of urbanization also presented the pattern of a multi-centre agglomeration and diffusion. Specifically, the regions of the highest internal coordination degree of urbanization were located in the Beijing-Tianjin-Hebei region, the Yangtze River Delta and the Pearl River Delta, with the regions of lower internal coordination degree of urbanization spreading around these three regions.

Third, the spatial distribution of the internal coordinated development of China's urbanization did not change on a large scale, but rather, changed in several small regions over the ten year period. The internal coordination degree of urbanization in the cities of northeastern China, Erdos in the Inner Mongolia Autonomous Region, Xinyang in the Henan Province, and Suizhou and Xiangyang in the Hubei Province gradually weakened, while the internal coordination degree of urbanization in the cities in the southwestern Shandong Province, northwestern Jiangsu Province and Urumqi and Karamay in the Xinjiang Autonomous Region strengthened over the ten years. The growth rate of the internal coordination degree of China's urbanization displayed a pattern of dotted distribution. 
The growth rate of the internal coordination degree in the cities of China's western provinces, such as Gansu, Xinjiang, Guizhou, and Sichuan, had clearly increased, and the growth rate of the western region was higher than that of the central and eastern regions.

Through the above analysis, it can be concluded that although population migration, economic growth and spatial expansion have made great contributions to China's urbanization development, internal contradictions have been increasing for a long time, such as the ecological environment deterioration, unbalanced public services and unreasonable spatial planning. Overall, there are a number of reasons why the internal coordination degree of urbanization is different across the 283 cities in China. Although the economic development and social services in the eastern region are better than those in other regions, the ecological environment problems, which last for a long time, result in an imbalanced development of urbanization. In addition to the above reasons, weak public services are another reason for the internal uncoordinated development of urbanization in the central region of China. The western region has advantages in terms of the ecological environment; however, urbanization levels in terms of economic development, population flow, social services and other aspects are relatively weak.

Therefore, China's urbanization development needs to be further adjusted according to the internal coordinated development of different regions. In the future, the development of urbanization in Beijing-Tianjin-Hebei Region, Yangtze River Delta and Pearl River Delta shall focus on accelerating the transformation of economic development mode, especially on circular economy mode, so as to achieve comprehensive improvements by constantly making up for their own deficiencies. As for the urbanization development of cities in Liaoning, Hebei, Shandong, Jiangsu, Zhejiang, Fujian and Guangdong provinces located around the above three regions, we shall not only pay attention to the speed and scale of urbanization development, but also strengthen the upgrading and promotion of basic public services within these regions, and focus more on the social and ecological effects of urbanization. The cities in the northeast and central provinces represented by Heilongjiang, Jilin, Shanxi, Henan, Hubei, Hunan and Jiangxi shall speed up the innovation of systems and mechanisms, gradually release the potential of urbanization development by orderly promoting the mobility of urban population, and focus on achieving the healthy development of urbanization by improving the equalization of urban and rural public services. The vast majority of cities in Western China shall further improve the spatial development potential for urbanization, accelerate the new-type industrialization development on the premise of quality assurance, and promote the development of urbanization systems into dense urban areas that agglomerate industry and population through improving economic development and attracting the concentration of human, financial and material resources.

This paper has some shortcomings, although it clearly explains the temporal and spatial distribution and the evolution of the coordinated development of urbanization in China. For example, due to the missing statistical data in 283 cities, the constructed indicator system lacks some important indicators, such as urban population ratio and PM 2.5, etc. However, the lack of these variables will not affect the credibility of the results in this study to a large extent. In addition, the discussion part of this paper is only carried out from a qualitative point of view. On the basis of this paper, further exploration of the driving forces that affect the coordinated development of urbanization in China from the perspective of econometric analysis will be an important part of future research.

Author Contributions: L.Z. conceived and designed this study, collected and analysed the data, and wrote the manuscript. Y.S. revised the manuscript. All authors read and approved the manuscript.

Funding: This research was funded by the National Natural Science Foundation of China, grant number [71573151], the China Postdoctoral Science Foundation, grant number [2017M620812, 2018M641399] and the Tsinghua University Initiative Scientific Research Program, grant number [2017THZWYX02].

Acknowledgments: The Department of Statistics at Beijing Forestry University made enormous contributions to our data analysis that is greatly appreciated.

Conflicts of Interest: The authors declare no conflict of interest. 


\section{References}

1. Shen, H.; Teng, F.; Song, J. Evaluation of spatial balance of China's regional development. Sustainability 2018, 10, 3314. [CrossRef]

2. Wang, Y.; Huang, H.; Shi, P. Spatial and temporal evolution of urbanization quality in China. Econ. Geogr. 2017, 1, 90-97. (In Chinese)

3. Qi, W.; Gao, Y.; Zhang, Q. Spatiotemporal dynamics of Beijing's urbanization efficiency from 2005 to 2014. Sustainability 2017, 9, 2190. [CrossRef]

4. Li, X.; Fu, S.; Lu, L. Study of the urbanization spatial differentiation of the Yangtze River economic belt. World Reg. Stud. 2017, 3, 84-95. (In Chinese)

5. Wang, X.; Qin, S.; Wu, N. Connotation, measurement of new-type urvanization and the characteristics of its spatial variation. Areal Res. Dev. 2014, 4, 69-75. (In Chinese)

6. Agesa, R. The incentive for rural to urban migration: A reexamination of the Harris-Todaro model. Appl. Econ. Lett. 2000, 7, 107-110. [CrossRef]

7. Behname, M. FDI localization, wage and urbanization in central Europe. Romanian Econ. J. 2013, 48, $23-36$.

8. Andersen, H.; Moller-Jensen, L.; Engelstoft, S. The end of urbanization? Towards a new urban concept or rethinking urbanization. Eur. Plan. Stud. 2011, 19, 595-611. [CrossRef]

9. Yu, W.; Zhang, P. Urbanization process, spatial spillover and green economic efficiency growth: A spatial econometric study based on provincial units from 2002 to 2012. Inq. Econ. Iss. 2016, 1, 77-82. (In Chinese)

10. Gabriel, S.A.; Rosenthal, S.S. Urbanization, agglomeration economies, and access to mortgage credit. Reg. Sci. Urban Econ. 2013, 43, 42-50. [CrossRef]

11. Maparu, T.; Mazumder, T. Transport infrastructure, economic development and urbanization in India (1990-2011): Is there any causal relationship. Transp. Res. Pt. A-Policy Pract. 2017, 100, 319-336. [CrossRef]

12. Lin, Y.; Ma, Z.; Zhao, K.; Hu, W.; Wei, J. The impact of population migration on urban housing prices: Evidence from China's major cities. Sustainability 2018, 10, 3169. [CrossRef]

13. Arvin, M.; Pradhan, R.; Norman, N. Transportation intensity, urbanization, economic growth, and $\mathrm{CO}_{2}$ emissions in the G-20 countries. Util. Policy 2015, 35, 50-66. [CrossRef]

14. Bao, C.; Chen, X. Review and prospect of research on the spatial pattern of China's urban system. Prog. Geogr. 2014, 10, 1300-1311. (In Chinese)

15. Yang, Y.; Liu, J.; Zhang, Y. An analysis of the implications of China's urbanization policy for economic growth and energy consumption. J. Clean. Prod. 2017, 161, 1251-1262. [CrossRef]

16. Park, H.; Fan, P.; John, R. Urbanization on the Mongolian Plateau after economic reform: Changes and causes. Appl. Geogr. 2017, 86, 118-127. [CrossRef]

17. Wang, Y.; Peng, B.; Xiong, J.; Wang, Q. The economy urbanization and population urbanization of Dongting Lake Area in China since 2001. Geogr. Sci. 2014, 1, 67-75. (In Chinese)

18. Brueckner, M. Economic growth, size of the agricultural sector, and urbanization in Africa. J. Urban Econ. 2012, 71, 26-36. [CrossRef]

19. de Andrés, M.; Barragán, J.; García Sanabria, J. Relationships between coastal urbanization and ecosystems in Spain. Cities 2017, 68, 8-17. [CrossRef]

20. Al-mulali, U.; Fereidouni, H.G.; Lee, J.Y.M.; Sab, C.N.B.C. Exploring the relationship between urbanization, energy consumption, and $\mathrm{CO}_{2}$ emission in MENA countries. Renew. Sust. Energ. Rev. 2013, 23, 107-112. [CrossRef]

21. Bakirtas, T.; Akpolat, A.G. The relationship between energy consumption, urbanization, and economic growth in new emerging-market countries. Energy 2018, 147, 110-121. [CrossRef]

22. Dhakal, S. GHG emissions from urbanization and opportunities for urban carbon mitigation. Curr. Opin. Environ. Sust. 2010, 2, 277-283. [CrossRef]

23. Liu, Y.; Zeng, C.; Cui, H.; Song, Y. Sustainable land urbanization and ecological carrying capacity: A spatially explicit perspective. Sustainability 2018, 10, 3070. [CrossRef]

24. Liu, N.; Liu, C.; Xia, Y. Examining the coordination between urbanization and eco-environment using coupling and spatial analyses: A case study in China. Ecol. Indic. 2018, 93, 1163-1175. [CrossRef]

25. He, J.; Wang, S.; Liu, Y. Examining the relationship between urbanization and the eco-environment using a coupling analysis: Case study of Shanghai, China. Ecol. Indic. 2017, 77, 185-193. [CrossRef] 
26. Wang, S.; Ma, H.; Zhao, Y. Exploring the relationship between urbanization and the eco-environment: A case study of Beijing-Tianjin-Hebei region. Ecol. Indic. 2014, 45, 171-183. [CrossRef]

27. Wan, L.; Ye, X.; Lee, J. Effects of urbanization on ecosystem service values in a mineral resource-based city. Habitat. Int. 2015, 46, 54-63. [CrossRef]

28. Churkina, N.; Zaverskiy, S. Challenges of strong concentration in urbanization: The case of Moscow in Russia. Procedia Eng. 2017, 198, 398-410. [CrossRef]

29. Cai, D.; Fraedrich, K.; Guan, Y.; Guo, S.; Zhang, C.; Zhu, X. Urbanization and climate change: Insights from eco-hydrological diagnostics. Sci. Total Environ. 2019, 647, 29-36. [CrossRef] [PubMed]

30. Shahbaz, M.; Sbia, R.; Hamdi, H. Economic growth, electricity consumption, urbanization and environmental degradation relationship in United Arab Emirates. Ecol. Indic. 2014, 45, 622-631. [CrossRef]

31. Shuaib, M.; Ali, K.; Ahmed, S.; Hussain, F.; Ilyas, M.; Hassan, N.; Khan, I.; Hussain, F. Impact of rapid urbanization on the floral diversity and agriculture land of district Dir, Pakistan. Acta. Ecol. Sin. 2018, 38, 394-400. [CrossRef]

32. Lin, B.; Zhu, J. Changes in urban air quality during urbanization in China. J. Clean. Prod. 2018, 188, 312-321. [CrossRef]

33. Li, Y.; Dai, Z.; Liu, X. Analysis of spatial-temporal characteristics of the PM 2.5 concentrations in Weifang City, China. Sustainability 2018, 10, 2960. [CrossRef]

34. Yang, D.; Ye, C.; Wang, X. Global distribution and evolvement of urbanization and PM 2.5 (1998-2015). Atmos. Environ. 2018, 182, 171-178. [CrossRef]

35. Lyu, H.; Wu, Y.; Shen, J.; Zhou, A. Assessment of social-economic risk of Chinese dual land use system using fuzzy AHP. Sustainability 2018, 10, 2451. [CrossRef]

36. Chen, J.; Guo, F.; Wang, H.; Wang, Z.; Wu, Y. Urban land revenue and sustainable urbanization in China: Issues and challenges. Sustainability 2018, 10, 2111. [CrossRef]

37. Lin, Y.; Li, Y.; Ma, Z. Exploring the interactive development between population urbanization and land urbanization: Evidence from Chongqing, China (1998-2016). Sustainability 2018, 10, 1741. [CrossRef]

38. Shannon, M.; Otsuki, K.; Zoomers, A.; Kaag, M. Sustainable urbanization on occupied land? The politics of infrastructure development and resettlement in Beira City, Mozambique. Sustainability 2018, 10, 3123. [CrossRef]

39. Ma, L.; Cheng, W.; Qi, J. Coordinated evaluation and development model of oasis urbanization from the perspective of new urbanization: A case study in Shandan County of Hexi Corridor, China. Sustain. Cities Soc. 2018, 39, 78-92. [CrossRef]

40. Zhu, F.; Zhang, F.; Li, C.; Jiao, P.; Wang, J. Coordination and regional difference of urban land expansion and demographic urbanization in China during 1993-2008. Prog. Geogr. 2014, 5, 647-656. (In Chinese)

41. Liu, H.; Deng, H.; Li, X. Research on the spatial and temporal difference of coordinated development between population urbanization and land urbanization in Yangtze River Economic Belt. China Popul. Resour. Environ. 2016, 5, 160-166. (In Chinese)

42. Guo, F.; Li, C.; Chen, C.; Gan, J. Spatial-temporal coupling characteristics of population urbanization and land urbanization in Northeast China. Econ. Geogr. 2015, 9, 49-56. (In Chinese)

43. Cui, X. Population urbanization and land urbanization in ethnic minority areas: Disequilibrium and spatial heterogeneity. China Popul. Resour. Environ. 2014, 8, 63-72. (In Chinese)

44. Xiong, X.; Xu, Y. Research on level and mechanical machine under the guidance of new urbanization. J. Quant. Tech. Econ. 2018, 2, 44-63. (In Chinese)

45. Cloke, P. Changing patterns of urbanization in rural areas of England and Wales, 1961-1971. Reg. Stud. 1978, 12, 603-617. [CrossRef] [PubMed]

46. Inkeles, A. Modernization and postmodernization: Cultural, economic, and political change in 43 societies. J. Democr. 1998, 9, 175-181. [CrossRef]

47. China International Urbanization Development Strategy Research Committee. China's Urbanization Quality Assessment System; China Social Sciences Academic Press: Beijing, China, 2013. (In Chinese)

48. Guo, Y. The essential connotation and evaluation index system of urbanization quality. Stud. Pract. 2013, 3, 13-20. (In Chinese)

49. Urban Socio-Economic Survey Team of China National Bureau of Statistics. Establishment and application of urbanization quality evaluation system in China. Stat. Res. 2005, 7, 15-19. (In Chinese) 
50. Zhang, Y.; Yang, Q.; Li, C.; Yang, M. The quality evaluation and comparative analysis of new urbanization development in Chongqing, China. Econ. Geo. 2015, 7, 79-86. (In Chinese)

51. Lv, D.; Ye, M.; Yang, Q. Review and reconstruction of new urbanization quality evaluation index system. Resear. Financ. Econ. Issues 2014, 9, 72-78. (In Chinese)

52. Fang, C.; Wang, Y. A comprehensive assessment of urban vulnerability and its spatial differentiation in China. Acta Geo. Sin. 2015, 2, 234-247. (In Chinese)

53. Haken, H. Advanced Synergetics; Springer: Berlin/Heidelberg, Germany; New York, NY, USA, 1983.

54. Haken, H. Synergetics Introduction and Advanced Topics; Springer: Berlin/Heidelberg, Germany, 2004.

55. Fan, J.; Kong, L.; Zhang, X. Synergetic effects of water and climate policy on energy-water nexus in China: A computable general equilibrium analysis. Energ. Policy 2018, 123, 308-317. [CrossRef]

56. Zhang, X.; Li, H. Urban resilience and urban sustainability: What we know and what do not know? Cities 2018, 72, 141-148. [CrossRef]

57. Wu, W.; Niu, S. Evolutional analysis of coupling between population and resource-environment in China. Procedia Environ. Sci. 2012, 12, 793-801. [CrossRef]

58. Zhang, W.; Wang, M. Spatial-temporal characteristics and determinants of land urbanization quality in China: Evidence from 285 prefecture-level cities. Sustain. Cities Soc. 2018, 38, 70-79. [CrossRef]

59. Ren, Y.; Lu, L.; Zhu, D. Urban spatial expansion pattern and mechanism of Yangtze River Delta Urban Agglomeration. Inq. Econ. Iss. 2018, 4, 90-98. (In Chinese)

60. Du, Y.; Sun, T.; Peng, J. Direct and spillover effects of urbanization on PM 2.5 concentrations in China's top three urban agglomerations. J. Clean. Prod. 2018, 190, 72-83. [CrossRef]

61. Bai, Y.; Deng, X.; Jiang, S.; Zhang, Q.; Wang, Z. Exploring the relationship between urbanization and urban eco-efficiency: Evidence from prefecture-level cities in China. J. Clean. Prod. 2018, 195, 1487-1496. [CrossRef]

62. Chen, Y.; Chang, K.; Han, F.; Karacsonyi, D.; Qian, Q. Investigating urbanization and its spatial determinants in the central districts of Guangzhou, China. Habitat Int. 2016, 51, 59-69. [CrossRef]

63. Dong, F.; Bian, Z.; Yu, B.; Wang, Y.; Zhang, S.; Li, J.; Su, B.; Long, R. Can land urbanization help to achieve $\mathrm{CO}_{2}$ intensity reduction target or hinder it? Evidence from China. Resour. Conserv. Recycl. 2018, 134, $206-215$. [CrossRef]

64. Hu, Y. Spatial distribution of comprehensive urbanization level in Anhui Province. Procedia Comput. Sci. 2018, 131, 401-408. [CrossRef]

65. Shen, L.; Zhang, Z.; Zhang, X.; Yan, H.; He, H. Measuring incoordination-adjusted sustainability performance during the urbanization process: Spatial-dimensional perspectives. J. Clean. Prod. 2017, 143, 731-743. [CrossRef]

66. Shannon, C.E. A mathematical theory of communication. Bell Syst. Tech. J. 1948, 27, 379-423. [CrossRef]

67. Velázquez Martínez, O.; Van Den Boogaart, K.G.; Lundstr öm, M.; Santasalo-Aarnio, A.; Reuter, M.; Serna-Guerrero, R. Statistical entropy analysis as tool for circular economy: Proof of concept by optimizing a lithium-ion battery waste sieving system. J. Clean. Prod. 2019, 212, 1568-1579. [CrossRef]

68. Arora, B.; Wainwright, H.M.; Dwivedi, D.; Vaughn, L.J.S.; Curtis, J.B.; Torn, M.S.; Dafflon, B.; Hubbard, S.S. Evaluating temporal controls on greenhouse gas (GHG) fluxes in an Arctic tundra environment: An entropy-based approach. Sci. Total Environ. 2019, 649, 284-299. [CrossRef] [PubMed]

69. Duan, Y.; Mu, H.; Li, N.; Li, L.; Xue, Z. Research on Comprehensive Evaluation of Low Carbon Economy Development Level Based on AHP-Entropy Method: A Case Study of Dalian. Energy Procedia 2016, 104, 468-474. [CrossRef]

70. Liu, W.; Jiao, F.; Ren, L.; Xu, X.; Wang, J.; Wang, X. Coupling coordination relationship between urbanization and atmospheric environment security in Jinan City. J. Clean. Prod. 2018, 204, 1-11. [CrossRef]

71. Cui, X.; Fang, C.; Liu, H.; Liu, X. Assessing sustainability of urbanization by a coordinated development index for an Urbanization-Resources-Environment complex system: A case study of Jing-Jin-Ji region, China. Ecol. Indic. 2019, 96, 383-391. [CrossRef]

(C) 2019 by the authors. Licensee MDPI, Basel, Switzerland. This article is an open access article distributed under the terms and conditions of the Creative Commons Attribution (CC BY) license (http:/ / creativecommons.org/licenses/by/4.0/). 